\title{
Sialic acid supplementation ameliorates puromycin aminonucleoside nephrosis in rats
}

\author{
Izabella ZA Pawluczyk1, Maryam G Najafabadi', Jeremy R Brown ${ }^{1}$, Alan Bevington ${ }^{1}$ and Peter S Topham²
}

Defects in sialylation are known to have serious consequences on podocyte function leading to collapse of the glomerular filtration barrier and the development of proteinuria. However, the cellular processes underlying aberrant sialylation in renal disease are inadequately defined. We have shown in cultured human podocytes that puromycin aminonucleoside (PAN) downregulates enzymes involved in sialic acid metabolism and redox homeostasis and these can be rescued by cotreatment with free sialic acid. The aim of the current study was to ascertain whether sialic acid supplementation could improve renal function and attenuate desialylation in an in vivo model of proteinuria (PAN nephrosis) and to delineate the possible mechanisms involved. PAN nephrotic rats were supplemented with free sialic acid, its precursor $\mathrm{N}$-acetyl mannosamine or the NADPH oxidase inhibitor apocynin. Glomeruli, urine, and sera were examined for evidence of kidney injury and therapeutic efficacy. Of the three treatment regimens, sialic acid had the broadest efficacy in attenuating PANinduced injury. Proteinuria and urinary nephrin loss were reduced. Transmission electron microscopy revealed that podocyte ultrastructure, exhibited less severe foot process effacement. PAN-induced oxidative stress was ameliorated as evidenced by a reduction in glomerular NOX4 expression and a downregulation of urine xanthine oxidase levels. Sialylation dysfunction was improved as indicated by reduced urinary concentrations of free sialic acid, restored electrophoretic mobility of podocalyxin, and improved expression of a sialyltransferase. These data indicate that PAN induces alterations in the expression of enzymes involved in redox control and sialoglycoprotein metabolism, which can be ameliorated by sialic acid supplementation possibly via its properties as both an antioxidant and a substrate for sialylation.

Laboratory Investigation (2015) 95, 1019-1028; doi:10.1038/labinvest.2015.78; published online 29 June 2015

Efficient functioning of the glomerular permselective barrier is thought to rely on the integrity of the anionic glycocalyx associated with the glomerular filter. Evidence suggests that the charge characteristics of this 'polyanion', are important for efficient plasma ultrafiltration. ${ }^{1}$ In its absence, normal foot process structure and filtration slit organization become distorted leading to the collapse of the filtration barrier $^{2-5}$ and leakage of proteins into the urine. A number of studies have demonstrated that the negatively charged sugar sialic acid is important for this function as its removal by infusion of sialidase or neutralisation of surface negative charge by polycations have resulted in podocyte effacement and proteinuria. ${ }^{6,7}$ Conversely, infusion of $\alpha 1$-acid glycoprotein, a sialylated acute phase protein, into experimental animals with puromycin aminonucleoside (PAN) nephrosis, improved podocyte ultrastructure. ${ }^{8}$ In some human proteinuric renal diseases, reduced glomerular sialylation is also accompanied by reduced sialic acid content at other sites, including red blood cells, suggesting a more widespread defect of sialylation. ${ }^{9}$ Despite the fact that alterations in the sialic acid content of the glomerular permselective barrier of PAN nephrotic rats have been recognized since the 1970s and 80 s, ${ }^{10-13}$ the mechanisms underlying these observations still remain incompletely understood.

The proteinuria associated with this model is thought to be initiated by reactive oxygen species (ROS) $)^{14}$ and antioxidant treatments have been shown to provide protection against PAN-induced alterations in podocyte foot process morphology. ${ }^{15}$ Possibly related to this is the fact that, in addition to its negative charge, sialic acid also has a unique $\alpha$-ketocarboxylic acid structure, which has been shown to chemically scavenge $\mathrm{H}_{2} \mathrm{O}_{2} \cdot{ }^{16}$ Consequently loss of sialic acid not only results in depletion of negative charge but also a source of local, inherent ROS scavenging activity.

We have recently shown that sialic acid was able to quench the oxidative burst of superoxide anions induced by PAN in

'Department of Infection, Immunity and Inflammation, University of Leicester, Leicester, UK and ²John Walls Renal Unit, Leicester General Hospital, Leicester, UK Correspondence: Dr IZA Pawluczyk, PhD, Department of Infection, Immunity and Inflammation, Maurice Shock Building, University of Leicester, Leicester LE1 9HN, UK. E-mail: izap1@le.ac.uk

Received 29 September 2014; revised 16 March 2015; accepted 27 March 2015 
cultured human podocytes. In addition, PAN induced the downregulation of sialyltransferases, an effect which was at least partially restored by sialic acid treatment. This effect was also mimicked by addition of exogenous superoxide dismutase. ${ }^{13}$ These data, therefore, suggest that there could be a link between aberrant sialylation and oxidative stress.

Dietary supplementation of the sialic acid precursor $\mathrm{N}$-acetyl mannosamine (ManNAc) has previously been shown to correct defective sialylation and improve the accompanying renal lesions in murine models of hereditary inclusion body myopathy. ${ }^{17,18}$ We hypothesize that dietary supplementation of PAN nephrotic rats with free sialic acid, given its antioxidant properties, may be a more effective treatment modality in a proteinuric model of kidney disease exhibiting both desialylation and oxidative stress.

\section{MATERIALS AND METHODS In Vivo Study}

PAN nephrosis was induced in $200 \mathrm{~g}$ male Sprague Dawley rats (Charles River) by i.p. injection of $10 \mathrm{mg}$ PAN (Sigma)/ $100 \mathrm{~g}$ body weight (day 1). Control rats received saline (vehicle). All rats were fed a standard chow and were given free access to drinking water, which was either tap water (A), or tap water supplemented with $1 \mathrm{mg} / \mathrm{ml}$ sialic acid (Carbosynth) (B), $1 \mathrm{mg} / \mathrm{ml} \mathrm{ManNAc} \mathrm{(Carbosynth;} \mathrm{C)} \mathrm{or}$ $2 \mathrm{mg} / \mathrm{ml}$ apocynin (Sigma; D). Each PAN-treated group contained eight rats (four rats per group for saline controls). Overnight urines $(18 \mathrm{~h}$ ) were collected on day 9 (peak proteinuria) by housing rats in metabolic cages. Prior to sacrifice by cervical dislocation aortic blood samples were taken under anesthesia. Kidneys were removed and processed for analysis. All animals were housed in standard conditions. All experimental procedures were conducted in accordance with the UK Animals (Scientific procedures) Act. Two PANtreated rats receiving tap water only were excluded as one died and one failed to respond to PAN.

\section{Glomerular Preparations}

Glomeruli were prepared by standard serial sieving of kidney cortex as previously described. ${ }^{19}$ Glomeruli were washed in Hank's balanced salt solution and resuspended in lysis buffer $(0.01 \mathrm{M} \beta$-glycerophosphate, $1 \mathrm{mM}$ EDTA, $1 \mathrm{mM}$ EGTA, $0.05 \mathrm{M}$ Tris- $\mathrm{HCl}, 1 \mathrm{mM}$ sodium orthovanadate, $1 \mathrm{mM}$ benzamidine, $0.2 \mathrm{mM}$ PMSF, $1 \mu \mathrm{g} / \mathrm{ml}$ pepstatin A, $1 \mu \mathrm{g} / \mathrm{ml}$ leupeptin, $0.1 \% \quad \beta$-mercaptoethanol, $1 \%$ Triton-X 100, $50 \mathrm{mM}$ sodium fluoride).

\section{Urine Analysis}

Whole urines were analyzed for urea, creatinine, and sialic acid concentrations. Urine sediments were analyzed by western blotting. Urines were corrected for excreted volume then $500 \mu \mathrm{l}$ aliquots were centrifuged for $30 \mathrm{~min}$ at 13000 r.p.m. at $4{ }^{\circ} \mathrm{C}$. The resulting pellets were resuspended in $80 \mu \mathrm{l}$ PBS.

\section{Urine Xanthine Oxidase activity}

$1.5 \mathrm{ml}$ aliquots of urine were centrifuged at 13000 r.p.m. for $2 \mathrm{~min}$. The resulting sediments were resuspended in $100 \mu \mathrm{l}$ xanthine oxidase assay activity buffer and sonicated with two $5 \mathrm{~s}$ bursts. Insoluble material was removed by centrifugation at 13000 r.p.m. for $2 \mathrm{~min}$. Aliquots of cleared supernatant $(50 \mu \mathrm{l})$ were analyzed using a Xanthine Oxidase Activity Assay Kit (Sigma) as directed in the manufacturer's protocol.

\section{Renal Function}

Proteinuria was assessed by measuring protein concentrations in whole urine using BioRad DC assay. Urea and creatinine concentrations in urine and serum were determined by QuantiChrome assays according to manufacturer's protocols.

\section{SDS-PAGE and Western Blotting}

Urine pellet lysates (sediments) or glomerular lysates $(30 \mu \mathrm{g})$ were mixed with an equal volume of reducing sample buffer, heated to $100^{\circ} \mathrm{C}$ for $5 \mathrm{~min}$, and resolved by SDS-PAGE on gels of appropriate pore size. Resolved proteins were electrophoretically transferred to nitrocellulose membranes. The membranes were blocked with $5 \%(\mathrm{w} / \mathrm{v})$ skimmed milk in Tris-buffered saline containing $0.05 \%$ Tween 20 (TTBS), and then incubated overnight at $4{ }^{\circ} \mathrm{C}$ with primary antibody. The membranes were washed with TTBS after which bound primary antibody was detected by incubation with the appropriate horseradish peroxidase-conjugated secondary antibody at room temperature for at least $2 \mathrm{~h}$. Immunoreactive proteins were detected by enhanced chemiluminescence (Super Signal West Pico, Pierce). Protein loading was normalized by reprobing the membranes with antibodies against $\alpha$-tubulin. Band intensities were quantified using a BioRad GS-700 imaging densitometer.

Antibodies: mouse anti-desmin, rabbit anti-podocalyxin, mouse anti-nephrin, rabbit anti-NOX4, rabbit anti-xanthine oxidase and rabbit anti-beta-galactoside alpha-2,3-sialyltransferase 3 (ST3Gal 3), anti-neuraminidase (neu)-3 (Santa Cruz Biotechnologies), biotinylated sambus nigra agglutinin (SNA), and biotinylated maakia amurensis (MALII; Vector laboratories), rabbit anti-beta-galactoside alpha-2,6-sialyltransferase 1 (ST6Gal 1), rabbit anti-uridine diphospho-Nacetylglucosamine 2-epimerase/ $\mathrm{N}$ acetyl mannosamine kinase (GNE; Biorbyt), rabbit anti- $\alpha$-tubulin (Cell Signalling Technology).

\section{Sialic Acid Levels}

Free and total sialic acid concentrations in whole urine or serum were measured using Quantichrome sialic acid assays.

\section{Electron Microscopy}

For transmission electron microscopy, small pieces of rat kidney cortex were fixed overnight in $2 \%$ gluteraldehyde $/ 4 \%$ formaldehyde in $0.1 \mathrm{M}$ cacodylate buffer with $2 \mathrm{mM}$ calcium chloride $(\mathrm{pH}=4)$ and then washed in the same cacodylate $/ \mathrm{CaCl}$ buffer. The tissue fragments were then fixed with $1 \%$ 
OsO4/1.5\% potassium ferricyanide for $2 \mathrm{~h}$, washed with distilled, de-iodized water and finally tertiary fixed with $1 \%$ uranyl acetate for $1 \mathrm{~h}$. The tissue was then serially dehydrated through ethanol and propylene oxide and embedded in Spurr's modified low-viscosity resin. Thin sections of $\sim 80 \mathrm{~nm}$ thickness were cut using a Reichert Ultracut $S$ ultramicrotome, collected onto copper mesh grids, and stained with Reynolds' lead citrate. The grids were viewed under a JEOL JEM-1400 electron microscope at $80 \mathrm{kV}$. Digital images were recorded using a SIS digital camera and iTEM software. The electron micrographs were then analyzed by a renal pathologist blinded to the treatments.

\section{Statistics}

Data are presented as means \pm s.e.m. Multiple comparisons between means were determined by an analysis of variance with Bonferroni correction. Statistical significance was defined as $P<0.05$.

\section{RESULTS}

\section{Kidney Function}

Dietary supplementation of PAN nephrotic rats demonstrated that sialic acid significantly reduced proteinuria by $31 \%$ $(P=0.03$; Figure 1$)$. However, no significant difference was observed between treatments.

All PAN-treated rats, regardless of supplementation, had raised serum creatinine concentrations $(P<0.009$; Figure 2a). In contrast, there was no difference in urine creatinine concentrations between supplements with the exception of the apocynin-treated rats that exhibited higher levels $(P<0.001$; Figure 2b).

All PAN-treated rats had raised serum urea levels compared with saline controls $(P \leq 0.001$; Figure $2 c)$. In contrast, there was no significant difference in urine urea concentrations between supplements with the exception of ManNAc rats, which exhibited lower levels than those of saline controls $(P=0.009$; Figure 2d).

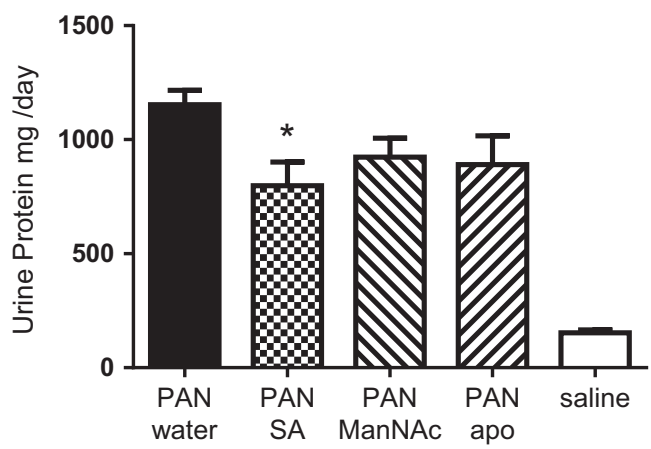

Figure 1 Effect of supplementation on PAN-induced proteinuria. Rat urine protein concentrations in $\mathrm{mg} / \mathrm{ml}$ were multiplied by urine volume and are presented as total protein excretion per day (means \pm s.e.m.). $\mathrm{PAN}_{\mathrm{SA}}$ rats exhibited reduced urine protein levels $\left({ }^{*} P<0.03\right.$ vs $\mathrm{PAN}_{\text {water; }}$; $n=6-16)$. apo, apocynin; ManNAc, N-acetyl mannosamine; PAN, puromycin aminonucleoside; SA, sialic acid.

\section{Podocyte Injury}

Podocyte injury or loss was determined by analyzing urine sediments for the slit diaphragm protein nephrin. Western blotting demonstrated significantly reduced nephrin levels in the urines of sialic acid supplemented rats $(P=0.008$; Figure 3).

Western blotting of glomerular lysates demonstrated that PAN treatment upregulated desmin, a marker of cytoskeletal damage often used as a marker of podocyte injury. ${ }^{20}$ Sialic acid supplementation significantly attenuated this effect more efficiently than the other supplements $(P=0.03$; Figure 4).

Transmission electron microscopy indicated that all PANtreated animals exhibited some degree of foot process effacement compared with saline controls. However, supplementation with sialic acid or apocynin reduced the extent of the damage compared with PAN-treated animals on water alone or supplemented with ManNAc (Figure 5a and e).

\section{Oxidative Stress}

As oxidative stress is considered to be the initiating trigger for podocyte damage in PAN nephrosis, western blotting was used to examine the expression of enzymes involved in redox control. PAN significantly raised glomerular expression of the NOX4 subunit of NADPH oxidase compared with saline controls $(P<0.003)$, while sialic acid significantly lowered PAN-induced expression $(P=0.007$; Figure 6a).

No difference in glomerular XO expression was observed between any of the rats regardless of treatment (data not shown). However, XO levels in urine were significantly elevated in PAN nephrotic rats compared with saline controls $(P<0.001$; Figure 6b). Both sialic acid and apocynin supplementation significantly reduced urine levels $(P<0.005$ and $P<0.001$, respectively). Analysis of $\mathrm{XO}$ activity in urines revealed that PAN-treated rat urines exhibited significantly raised activity levels compared with saline controls $(P<0.002$; Figure 6c). Urines from sialic acid and apocynin supplemented rats exhibited less $\mathrm{XO}$ activity than PAN- treated rats on water alone $(P<0.02$ and $P<0.03$, respectively).

\section{Sialylation}

Raised serum and urine sialic acid levels accompanied by tissue desialylation are usually a harbinger of an underlying problem with sialic acid metabolism. Analysis of urines and sera demonstrated that sialic acid and apocynin supplementation significantly reduced free sialic acid levels in the urines of PAN nephrotic rats $(P<0.03$ and $P<0.04$, respectively; Figure $7 \mathrm{a})$. None of the supplements reduced free or total sialic acid levels in serum (Figure $7 \mathrm{c}$ and $\mathrm{d}$ ).

To examine the effects of supplementation on the sialylation status of glomerular proteins, glomerular lysates were examined for the expression of sialoglycoproteins expressing sialic acid residues in $\alpha 2-6$ or $\alpha 2-3$ linkage using lectins SNA and MALII, respectively. Western blotting revealed two major sialylated bands with molecular weights 

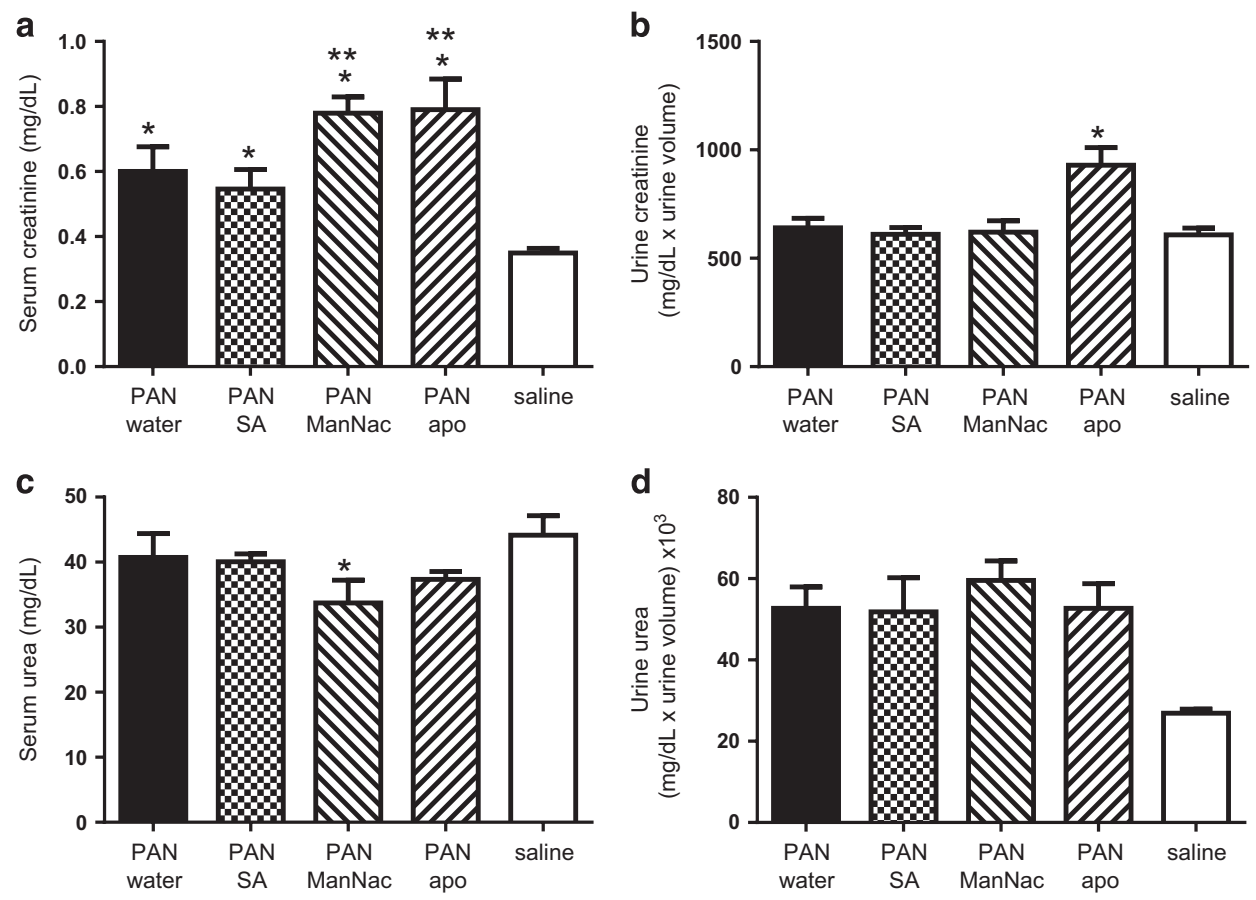

Figure 2 Effect of supplementation on urea and creatinine levels. Rat sera and urines were assayed for creatinine and urea. Data are expressed as $\mathrm{mg} / \mathrm{dL}$ for serum concentrations and $\mathrm{mg} / 18 \mathrm{~h}\left(\mathrm{mg} / \mathrm{dl} / 100 \times\right.$ urine volume) for urine levels. (a) Serum creatinine: ${ }^{*} P<0.009$ for all PAN-treated $v s$ controls; ${ }^{\dagger} P<0.006$ for PAN $_{\text {ManNAc }}$ and PAN apo $_{\text {vs }} \mathrm{PAN}_{\mathrm{SA}}$. (b) Urine creatinine: ${ }^{*} P<0.009$ for PAN $\mathrm{N}_{\mathrm{apo}}$ vs other treatments. (c) Serum urea: ${ }^{*} P<0.001$ for all PAN-treated vs controls. (d) Urine urea: n.s. between treatments $(n=6-16)$. apo, apocynin; ManNAc, N-acetyl mannosamine; PAN, puromycin aminonucleoside; SA, sialic acid.

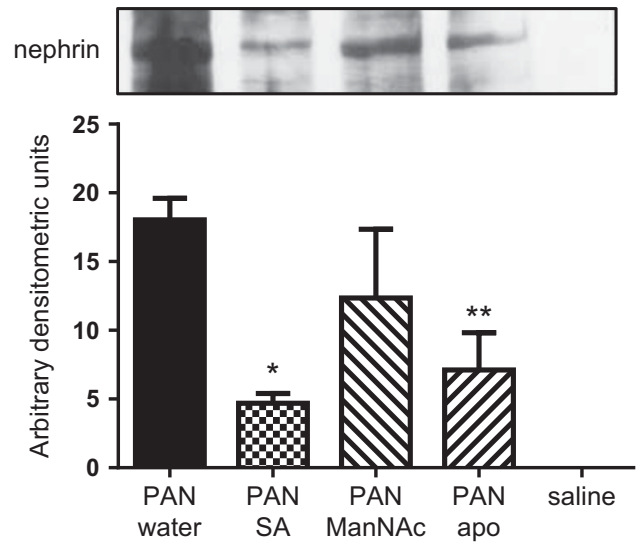

Figure 3 Effect of supplementation on urine nephrin. Rat urines were diluted to correct for excreted volume. Equal volumes were then centrifuged to generate sediment pellets, which were analyzed by western blotting for the podocyte specific protein nephrin. Western blot shows reduced urine nephrin loss in $\operatorname{PAN}_{S A}\left(* P=0.008\right.$ vs PAN $\left._{\text {water }}\right)$ and in $\mathrm{PAN}_{\text {apo }}\left({ }^{* *} P<0.03\right.$ vs $\left.\mathrm{PAN}_{\text {water }}\right)$. Representative blot is shown. Histogram shows densitometric data $(n=4)$. apo, apocynin; ManNAc, N-acetyl mannosamine; PAN, puromycin aminonucleoside; SA, sialic acid.

of the order of $125 \mathrm{kD}$ and $65 \mathrm{kD}$ (other minor bands were visible after longer exposure). PAN treatment significantly reduced the intensity of the $65 \mathrm{kD}$ band $(P<0.002$ and $P<0.04$ for SNA and MALII, respectively; Figure 8a).

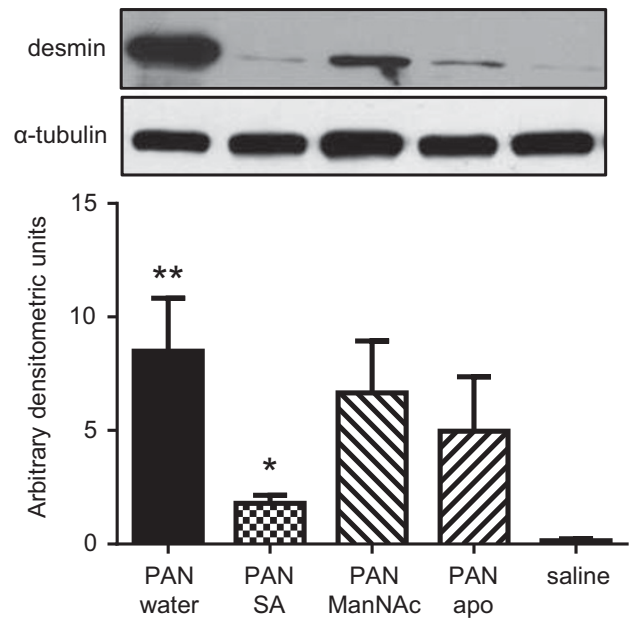

Figure 4 Effect of supplementation on glomerular desmin expression. Western blot shows increased glomerular desmin expression in $\mathrm{PAN}_{\text {water }}$ $\left({ }^{*} P<0.02\right.$ vs control), and reduced expression in $\operatorname{PAN}_{S A}\left({ }^{*} P=0.03\right.$ vs $\mathrm{PAN}_{\text {water }}$ ). Representative blot is shown. Histogram shows densitometric data $(n=5-8)$. apo, apocynin; ManNAc, $\mathrm{N}$-acetyl mannosamine; PAN, puromycin aminonucleoside; SA, sialic acid.

Although the lectin-binding westerns illustrated PANinduced changes in sialylation and their subsequent rescue as a result of supplementation, identification of the bands was beyond the scope of the study. 

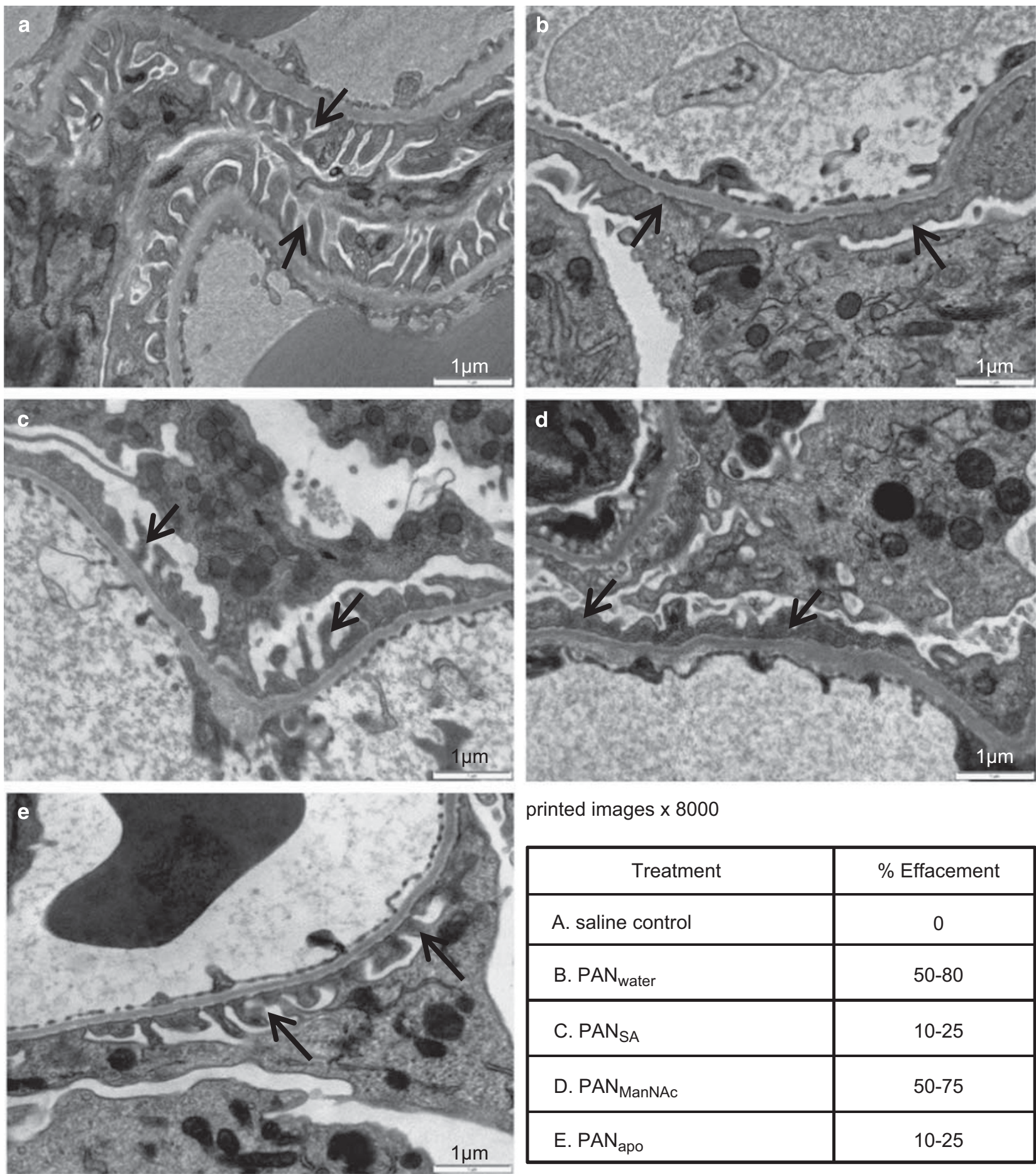

printed images $\times 8000$

\begin{tabular}{|c|c|}
\hline Treatment & $\%$ Effacement \\
\hline A. saline control & 0 \\
\hline B. $\mathrm{PAN}_{\text {water }}$ & $50-80$ \\
\hline C. PAN $_{S A}$ & $10-25$ \\
\hline D. PAN $\mathrm{ManNAc}_{\mathrm{c}}$ & $50-75$ \\
\hline E. $\mathrm{PAN}_{\mathrm{apo}}$ & $10-25$ \\
\hline
\end{tabular}

Figure 5 Effect of supplementation on podocyte structure. Transmission electron micrographs. (a) Saline-treated controls, (b) PAN water, (c) PAN $\mathrm{PA}_{\mathrm{SA}}$

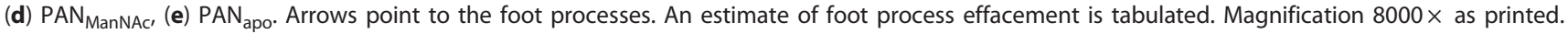
apo, apocynin; ManNAc, N-acetyl mannosamine; PAN, puromycin aminonucleoside; SA, sialic acid.

Western blotting of rat urine sediments with SNA demonstrated that sialic acid supplementation result in the excretion of fewer $\alpha 2-6$-sialylated glycoproteins than PAN rats on other supplements (Figure 8b). MALII staining indicated that $\alpha 2-3$-sialylated glycoproteins were largely detected in the urines of those rats receiving tap water or ManNAc (Figure 8b).

Podocalyxin is a highly sialylated podocyte protein known to have an important role in the maintenance of foot processes. ${ }^{21,22}$ Western blotting of glomerular lysates from 
PAN-treated rats demonstrated retarded mobility of podocalyxin on SDS-PAGE-a phenomenon consistent with a change in charge and glycosylation. Sialic acid

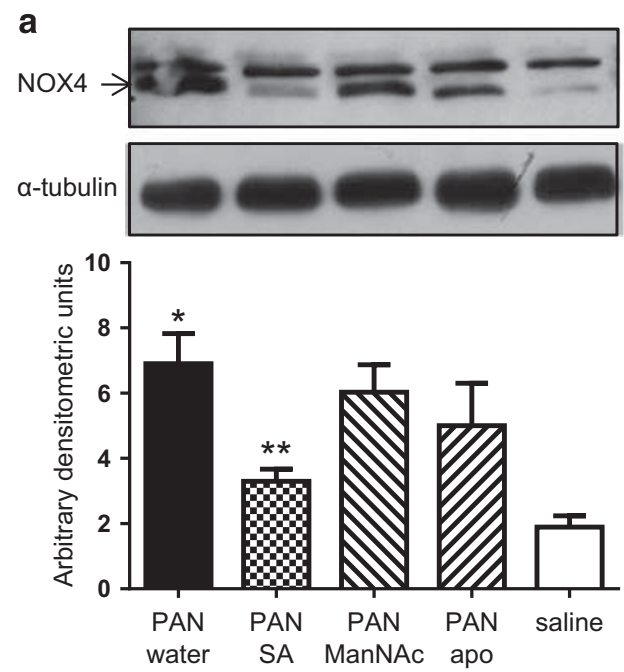

b

Xanthine oxidase - urine
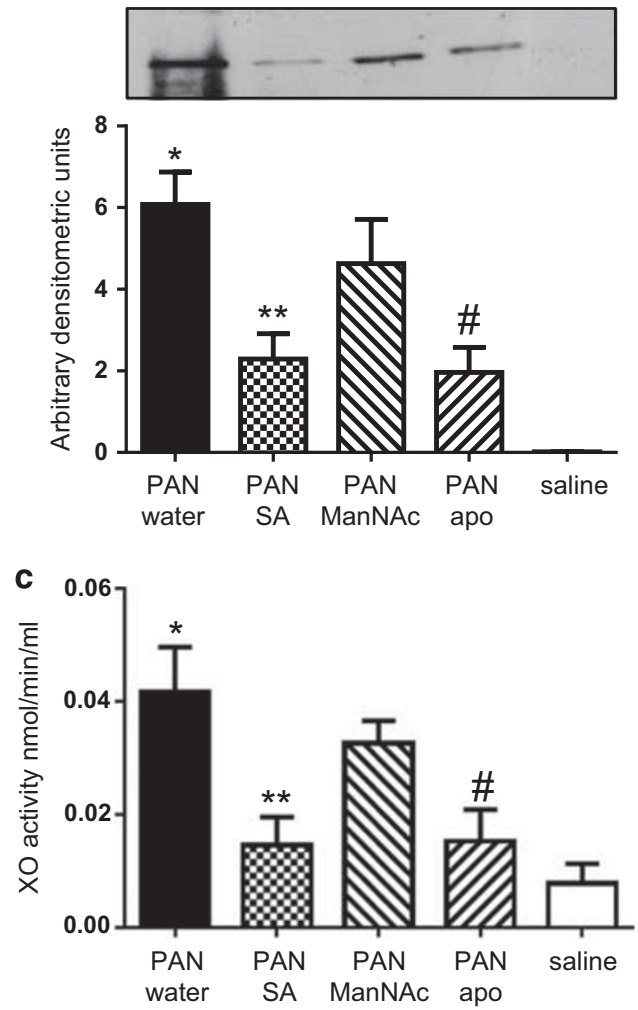

Figure 6 Effect of supplementation on oxidative enzymes. (a) Western blot shows increased glomerular NOX4 expression in $\mathrm{PAN}_{\text {water }}\left({ }^{*} P<0.003\right.$ vs saline) and reduced expression in $\operatorname{PAN}_{\mathrm{SA}}{ }^{* * P}=0.007$ vs $\mathrm{PAN}_{\text {wateri }}$ $n=4-8)$. (b) Western blot shows increased urine XO levels in PAN water $\left({ }^{*} P<0.001\right.$ vs control) and decreased levels in $\mathrm{PAN}_{\mathrm{SA}}\left({ }^{* *} P<0.005\right.$ vs $\left.\mathrm{PAN}_{\text {water }}\right)$ and PAN $\mathrm{Papo}\left({ }^{\#} P=0.001\right.$ vs $\left.\mathrm{PAN}_{\text {wateri }} n=5-8\right)$. (c) Histogram shows $X O$ activity derived from solubilised urine sediments $\left({ }^{*} P<0.02\right.$ vs $\mathrm{PAN}_{\text {water, }}{ }^{* *} P<0.03$ vs $\mathrm{PAN}_{\text {water, }}{ }^{\#} P<0.002$ vs saline control; $n=6$ ). apo, apocynin; ManNAc, $\mathrm{N}$-acetyl mannosamine; PAN, puromycin aminonucleoside; SA, sialic acid. supplementation appeared to correct the apparent change in electrophoretic mobility (Figure 8c).

In order to determine the cause of the altered sialylation, the expression of a number of enzymes involved in sialic acid metabolism was investigated. Immunoblotting indicated that GNE, the rate limiting enzyme of the sialic acid biosynthetic pathway was significantly upregulated in PAN-treated rats compared with saline controls $(P<0.03)$. Apocynin supplementation significantly reduced this upregulation $(P<0.03$; Figure 9).

Sialyltransferases catalyse the transfer of CMP-activated sialic acid residues onto glycoproteins in the Golgi apparatus. Western blotting demonstrated that PAN treatment significantly reduced the expression of sialyltransferase ST6Gal 1 and $(P<0.02)$. Both sialic acid and apocynin supplementation at least partially restored expression levels (Figure 10a). In contrast, expression of ST3Gal 3 was upregulated by PAN. However, only apocynin supplementation significantly restored levels closer to those of control (Figure 10b).

Reduced sialic acid levels can also occur as a result of excision. Western blotting for neu-3, the sialidase that cleaves sialic acid from renal gangliosides showed no difference in expression between any of the treatments (data not shown).

\section{DISCUSSION}

The results of this study have demonstrated that sialic acid supplementation is able to ameliorate the effects of PANinduced injury by reducing glomerular damage and subsequent proteinuria more effectively than apocynin or ManNAc. However, it must be stressed that although proteinuria was reduced, sialic acid supplementation had no effect upon serum creatinine levels that remained raised. As well as reducing podocyte effacement, sialic acid supplementation altered the pattern of podocyte protein expression in the glomerular lysates and urines of PAN nephrotic rats. More importantly, sialic acid was able to restore the expression of some of the enzymes involved in sialic acid metabolism and redox homeostasis.

Raised serum and urine sialic acid levels are usually a harbinger of an underlying defect of sialic acid metabolism. $^{23-25}$ Although PAN-elevated serum sialic acid levels were not modified by dietary supplementation, urinary excretion of free sialic acid was significantly reduced, indicating some degree of sialylation restoration.

As aberrant sialylation can occur as a result of a dysfunction in any one of a number of pathways involved in the production sialoglycoconjugates, we examined the expression of some of the enzyme involved in this process. GNE is the ubiquitously expressed, rate limiting enzyme of sialic acid biosynthesis. We observed an upregulation of GNE expression that could imply an increased through-put of sialic acid, perhaps in an attempt to correct reduced sialylation. However, in contrast to the in vivo data, we observed reduced expression of GNE in response to PAN in cultured human podocytes, an effect that was not rescued by sialic acid 

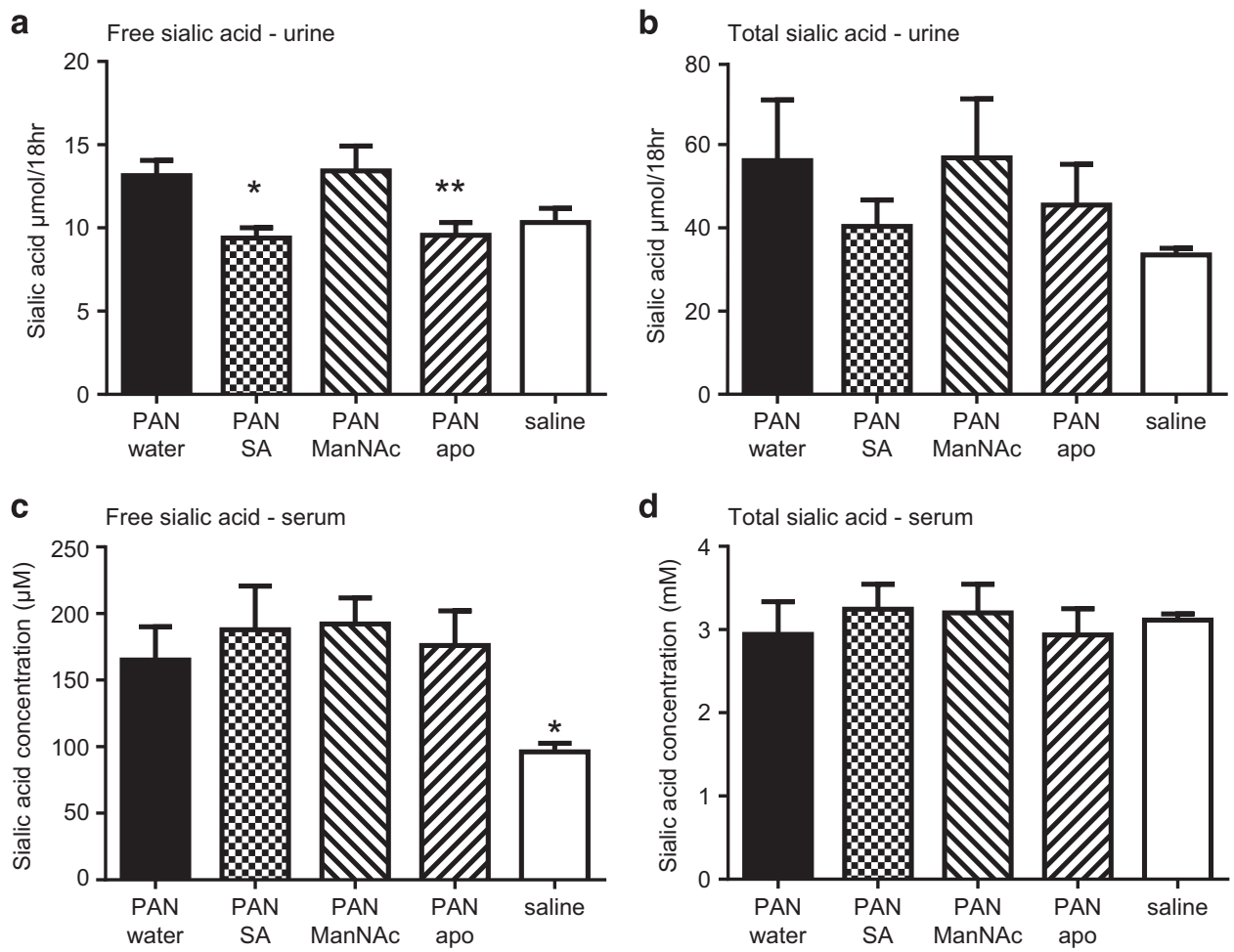

Figure 7 Effect of supplementation on free and total sialic acid levels. Rat sera and urines were analysed for free and total sialic acid. Data were expressed as $\mu \mathrm{M}$ or $\mathrm{mM}$ for serum and $\mu \mathrm{mol} / 18 \mathrm{~h}\left(\mu \mathrm{M} / 1000 \times\right.$ urine volume) for urine. (a) Urine 'free' sialic acid ${ }^{*} P<0.03$ for PAN ${ }_{S A} v s \mathrm{PAN}_{\text {water, }}$ and ${ }^{* *} p<0.04$ for PAN apo $_{\text {vs }} \mathrm{PAN}_{\text {water. }}$ (c) Serum 'free' sialic acid ${ }^{*} P<0.04$ for all PAN rats vs controls. (d, b) Serum and urine total sialic acid: $n . s(n=6-16)$. apo, apocynin; ManNAc, N-acetyl mannosamine; PAN, puromycin aminonucleoside; SA, sialic acid.

supplementation. ${ }^{13}$ It is now generally considered that cells have an excess of GNE beyond the amount required to supply flux into the sialic acid pathway, implying that GNE, as well as being involved in sialic acid biosynthesis, has other noncanonical functions. ${ }^{26,27}$ One might, therefore, surmise that the PAN-induced increase in GNE does not directly impact on its synthetic role but may modulate other non-canonical signaling pathways and genes involved in kidney injury.

Studies in other organ systems have shown that desialylation often occurs as a result of altered expression of sialyltransferases. ${ }^{28,29}$ This study demonstrated that PAN modulated the glomerular expression of sialyltransferases. In this case, however, apocynin proved to be a better restorer of expression than sialic acid. Our previous in vitro investigations demonstrated that the PAN-induced reduction in expression of sialytransferases in cultured human podocytes was improved by sialic acid treatment, an effect which was also mimicked by treatment with the enzyme superoxide dismutase $^{30}$ These data would suggest that the PAN-induced alterations in sialyltransferase expression are modulated by the oxidative milieu and could explain why sialic acid supplementation, by virtue of its antioxidant capacity, can restore some expression.

Reduced sialylation can also arise as a result of the actions of neuraminidases. We found that neu-3, a sialidase that cleaves sialic acid from gangliosides, was not significantly affected by PAN treatment (data not shown). These data concur with the observations of others who also failed to detect changes in sialidase activity in PAN nephrotic rats, despite the loss of glomerular sialic acid. ${ }^{31}$

Eguchi $e a^{32}$ have proposed that desialylation can occur as a direct result of the actions of ROS with the terminal sialic acid residues of oligosaccharide side chains. They suggested that the superoxide anion and related ROS specifically attack the carboxyl group of the terminal sugar leading to the cleavage of the glycosidic link and liberating the sialic acid residue from cell surface oligosaccharides. ${ }^{32}$

PAN nephrosis has long been thought to be initiated by oxidative podocyte damage ${ }^{14,33-35}$ and a number of studies have described the protective effects of antioxidants in this experimental model. $15,36,37$ However, no studies have yet described the effects of free sialic acid on PAN-induced redox alterations. As puromycin is metabolized to xanthine by glomerular enzymes, we examined the xanthine oxidase pathway to ascertain whether it was involved in the initiation of oxidative podocyte damage. Although we found no evidence of altered glomerular XO expression, which was in accordance with the observations of Ginevri et al, ${ }^{33}$ we did observe changes in enzyme protein and activity levels in the urine of PAN-treated rats. Although XO is generally thought 
a
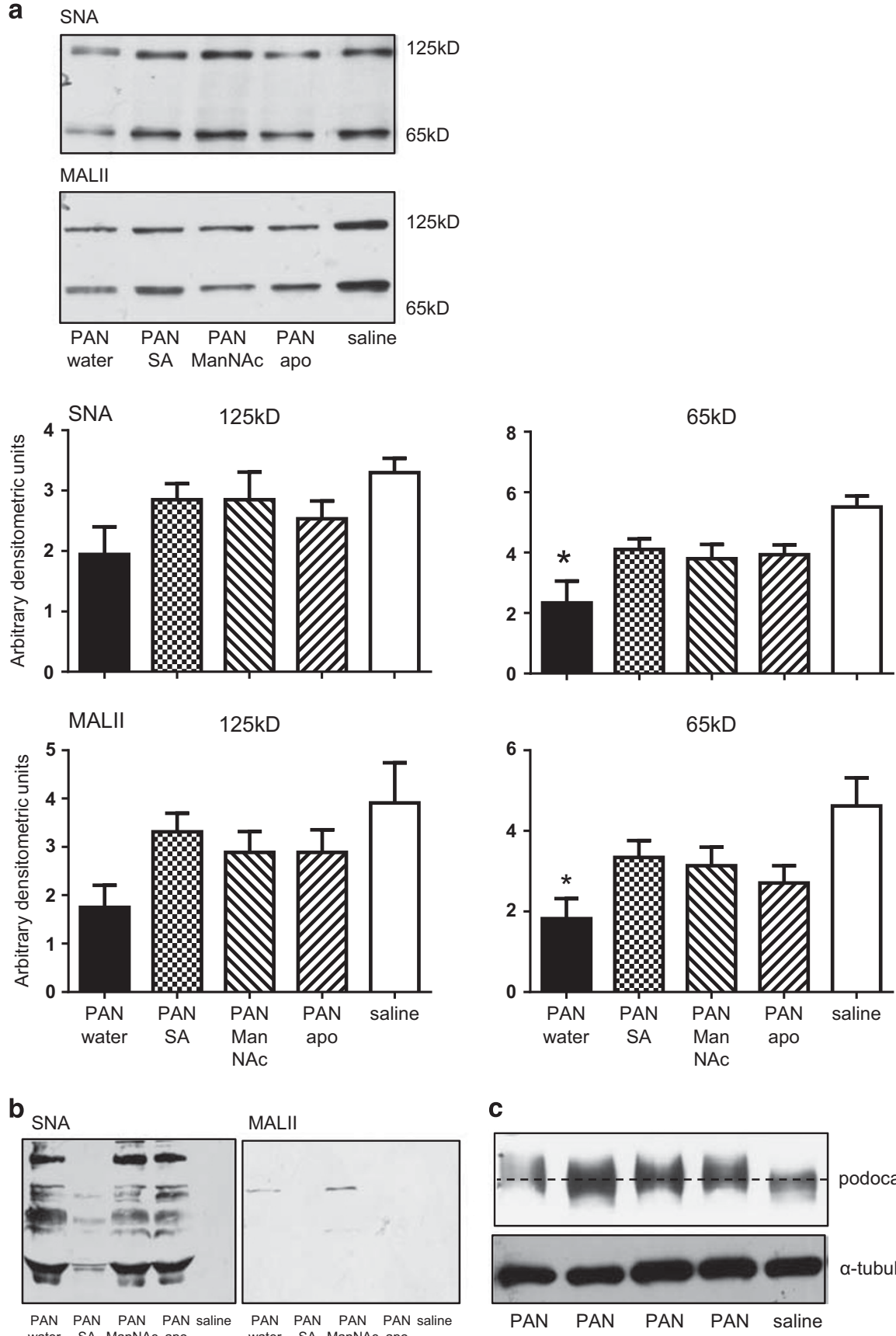

MALII

c

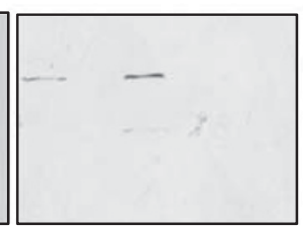

water SA ManNAc apo

PAN PAN PAN PAN
water SA ManNAc apo

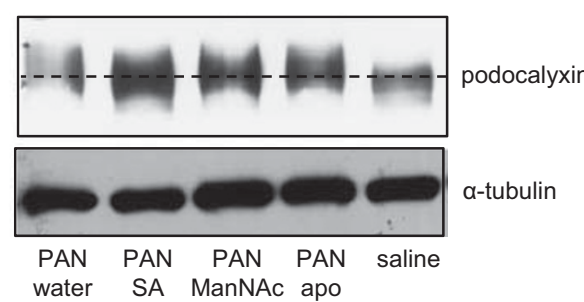

Figure 8 Effect of supplementation on glycoprotein sialylation. (a) Western blots showing patterns of glomerular a2-6 (SNA) and a2-3 (MALII) staining. The $65 \mathrm{kD}$ SNA stained band was reduced in intensity in $\mathrm{PAN}_{\text {water }}\left({ }^{*} P<0.002\right.$ vs saline). The $65 \mathrm{kD}$ MALIl stained band was reduced in PAN ${ }_{\text {water }}\left({ }^{*} P<0.04\right.$ vs saline). (b) Western blots of urine sediments show variations in SNA and MALIl staining. (c) Western blot shows podocalyxin staining in glomerular lysates. Dotted line represents the central position of podocalyxin in controls. Representative blots are shown, histograms show densitometric data $(n=4-8)$. apo, apocynin; ManNAc, N-acetyl mannosamine; PAN, puromycin aminonucleoside; SNA, sambus nigra agglutinin; SA, sialic acid.

to be an oxidative enzyme, what is much less appreciated is that uric acid, a product of XO activity, is also a potent ROS scavenger. ${ }^{38}$ The antioxidant capacity in serum strongly correlates with urate levels. ${ }^{38}$ Therefore, in the context of PAN nephrosis, loss of $\mathrm{XO}$, seen as increased urine xanthine oxidase, could be consistent with an injurious, oxidative effect, as it potentially results in reduced levels of a potent serum antioxidant.

$\mathrm{NADPH}$ oxidase is generally considered to be a major source of non-phagocytic cell superoxide anion production. ${ }^{39}$ 

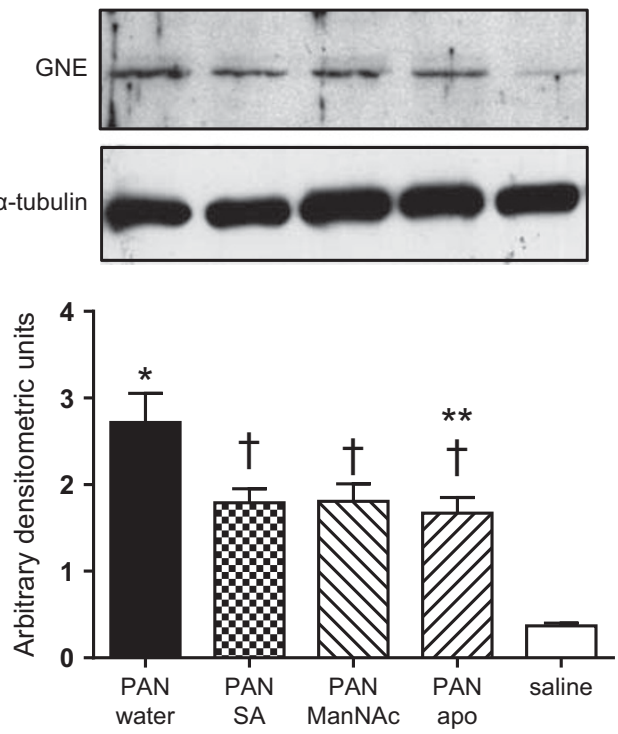

Figure 9 Effect of supplementation on GNE expression. Western blots of glomerular lysates show increased expression of GNE in PAN-treated rats (PAN $_{\text {water }}{ }^{*} P<0.001$ vs controls, $\mathrm{PAN}_{S \mathrm{SA}}, \mathrm{PAN}_{\mathrm{ManNA}}$, and $\mathrm{PAN}_{\text {apo }}{ }^{\dagger} P<0.03$ vs control), $\mathrm{PAN}_{\mathrm{SA}}{ }^{*} P<0.03$ vs $\mathrm{PAN}_{\text {water }}$. Apocynin reduced $\mathrm{PAN}$-induced upregulation ( $* * P<0.03$ ). Representative blot is shown. Histogram shows densitometric data $(n=3-7)$. apo, apocynin; GNE, diphospho-Nacetylglucosamine 2-epimerase/ $\mathrm{N}$ acetyl mannosamine kinase; ManNAc, $\mathrm{N}$-acetyl mannosamine; PAN, puromycin aminonucleoside; SA, sialic acid.

Sialic acid supplementation downregulated the expression of the PAN-induced NOX4 subunit and appeared to do this more efficiently than apocynin. But this may be due to the fact that apocynin exerts its effects by inhibiting the assembly of enzymic subunits rather than by reducing expression.

Whether oxidative stress and desialylation are causally related in renal disease is yet to be unequivocally established, although the results presented here together with those of our previous in vitro study suggest this could be the case. Furthermore, oxidative stress-related desialylation has previously been reported in association with Dengue fever ${ }^{40}$ and hyperthyroidism. ${ }^{41}$

Of the three supplements, sialic acid proved to have the broadest spectrum of efficacy, although a number of the beneficial effects were also common to apocynin. Again this would imply that in those parameters, the effects of sialic acid were largely mediated via its properties as an antioxidant. Despite the efficacy of ManNAc in GNE myopathy, ${ }^{17,18}$ its benefits as a therapeutic supplement in this study were not comparable to those of sialic acid. In experimental murine GNE myopathy, desialylation occurs as a consequence of a specific mutation in one enzyme of the sialic acid biosynthetic pathway, which although lethal, is adequately bypassed using ManNAc as a substrate (and also notably sialic acid ${ }^{42}$ ). However, the prevailing oxidative conditions encountered in PAN nephrosis clearly affect the expression of a number of enzymes, any of which could affect the phenotype of the disease. The efficacy of sialic acid supplementation under
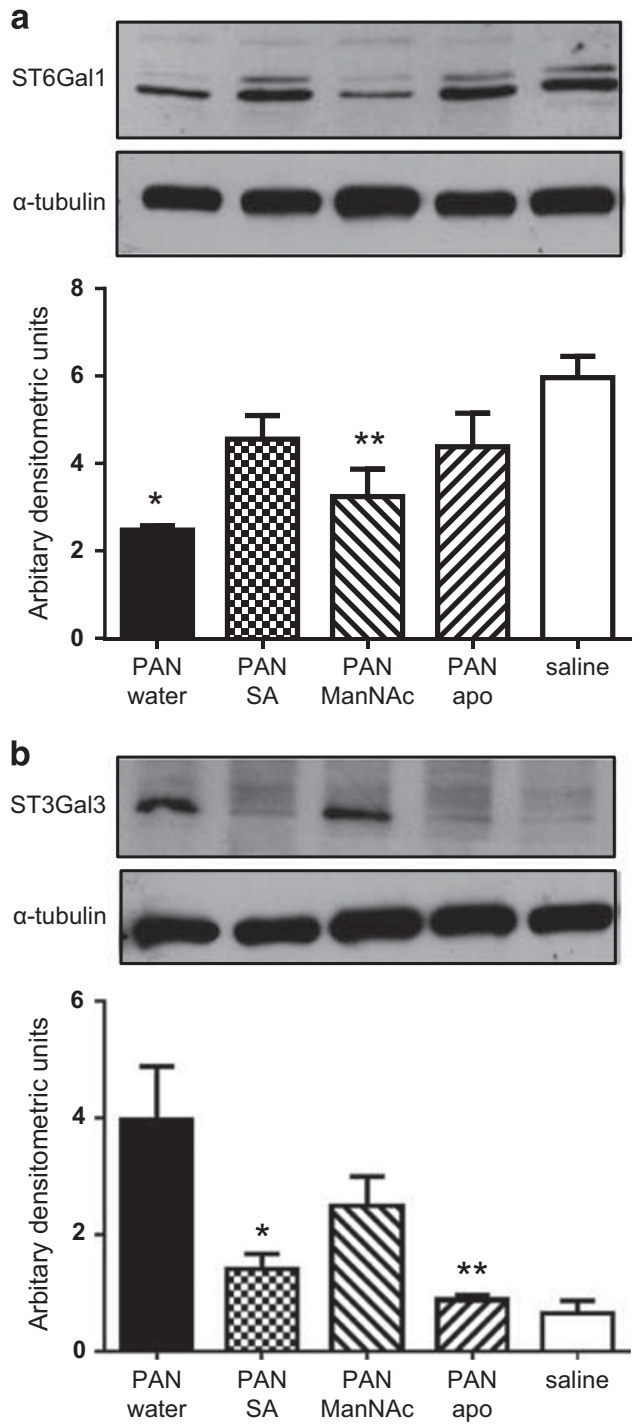

Figure 10 Effect of supplementation on sialyltransfersase expression. Western blots of glomerular lysates show (a) decreased ST6Gal 1 expression in $\mathrm{PAN}_{\text {water }}$ and $\mathrm{PAN}_{\text {ManNAc }}\left({ }^{*} P<0.01\right.$ and ${ }^{* *} P<0.02$ vs saline, respectively; $n=4-8)$ (b) increased ST3Gal 3 expression in $\mathrm{PAN}_{\text {water }}$ ( ${ }^{*} P<0.005$ vs saline), and decreased expression in $\mathrm{PAN}_{\text {apo }}\left({ }^{*} P<0.04\right.$ vs $\left.\mathrm{PAN}_{\text {water }} ; n=5-8\right)$. Representative blots are shown. Histograms show densitometric data. apo, apocynin; ManNAc, N-acetyl mannosamine; PAN, puromycin aminonucleoside; ST3Gal 3, alpha-2,3-sialyltransferase 3; ST6Gal 1, alpha-2,6-sialyltransferase 1; SA, sialic acid.

these conditions must therefore lie in its additional property as an antioxidant.

It would have been illuminating to carry out activity assays on all of the enzymes of sialic acid metabolism and redox control investigated in this study in addition to measuring expression levels. However, the amount of glomerular tissue generated did not allow for this and therefore this remains a drawback of the study.

In conclusion, this study has illustrated that a number of enzymes involved in sialic acid metabolism and redox control 
are affected by PAN treatment, the expression of some can be rescued by sialic acid supplementation. This study provides evidence to support further investigation of sialic acid as a therapeutic modality in proteinuric renal disease.

\section{ACKNOWLEDGMENTS}

We would like to acknowledge Stefan Hyman and Natalie Allcock of the University of Leicester's electron microscopy suite for their advice and assistance with the electron microscopy. We would also like to thank Professor Peter Furness for help with analyzing the transmission electron micrographs. This study was supported by a grant from Kidney Research UK grant number [RP31/2011].

\section{DISCLOSURE/CONFLICT OF INTEREST}

The authors declare no conflict of interest.

1. Mohos SC, Skoda L. Glomerular sialoproteins. Science 1969;164: 1591-1596.

2. Jones DB. Mucosubstances of the glomerulus. Lab Invest 1969;21: 119-125.

3. Michael AF, Blau E, Vernier RL. Glomerular polyanion: alteration in aminonucleoside nephrosis. Lab Invest 1970;23:649-657.

4. Holthofer H, Virtanen I, Pettersson E et al. Lectins as fluorescence microscopic markers for saccharides in the human kidney. Lab Invest 1981;45:391-399.

5. Murata F, Tsuyama S, Suzuki S et al. Distribution of glycoconjugates in the kidney studied by use of labelled lectins. J Histochem Cytochem 1983;31:139-144.

6. Gelberg H, Healy L, Whiteley $\mathrm{H}$ et al. In vivo removal of alpha 2-6 linked sialic acid from the glomerular filtration barrier results in podocyte charge alteration and glomerular injury. Lab Invest 1996;74:907-920.

7. Firth JD. Effect of polycations on the function of the isolated perfused kidney. Clin Sci 1990;79:591-598.

8. Muchitsch E-M, Pichler L, Schwarz HP et al. Effects of human alpha-1acid glycoprotein on aminonucleoside-induced minimal change nephrosis in rats. Nephron 1999;81:194-199.

9. Boulton-Jones JM, McWilliams G, Chandrachud L. Variation in charge on red cells of patients with different glomerulopathies. Lancet 1986;2: 186-189.

10. Michael AF, Blau E, Vernier RL. Glomerular polyanion. Alteration in aminonucleoside nephrosis. Lab Invest 1970;23:649-657.

11. Caulfield J-P, Farquar MG. Loss of anionic sites from the glomerular basement membrane in aminonucleoside nephrosis. Lab Invest 1978;39:505-512.

12. Kerjaschki D, Vernillo T, Farquhar MG. Reduced sialylation of podocalyxin - the major sialoprotein of the rat kidney glomerulus in aminonucleoside nephrosis. Am J Pathol 1985;118:343-349.

13. Quatacker J. Alterations in the sialic acid content of the rat glomerular filter in aminonucleoside nephrosis. Virchows Arch B cell Pathol Incl Mol Pathol 1986;50:237-247.

14. Diamond JR, Bonventre JV, Karnovsky MJ. A role for free radicals in aminonucleoside nephrosis. Kidney Int 1986;29:478-483.

15. Ricardo SD, Bertram JF, Ryan GB. Antioxidants protect podocyte foot processes in puromycin aminonucleoside-treated rats. J Am Soc Nephrol 1986;4:1974-1986.

16. lijima R, Takahashi $H$, Namme $R$ et al. Novel biological function of sialic acid ( $\mathrm{N}$-acetylneuraminic acid) as a hydrogen peroxide scavenger. FEBS Lett 2004;561:163-166.

17. Galeano B, Klootwijk R, Manoli I et al. Mutation in the key enzyme of sialic acid biosynthesis causes severe glomerular proteinuria and is rescued by N-acetylmannosamine. J Clin Invest 2007;117:1585-1594.

18. Niethamer TK, Yardeni T, Leoyklang $\mathrm{P}$ et al. Oral monosaccharide therapies to reverse renal and muscle hyposialylation in a mouse model of GNE myopathy. Mol Gen Met 2012;107:748-755.

19. Foidart JB, Dechenne CA, Mahieu P et al. Tissue culture of normal glomeruli: Isolation and morphological characterisation of two homogenous cell lines. Invest Cell Pathol 1979;2:15-26.
20. Yaoita E, Kawasaki K, Yamamoto T et al. Variable expression of desmin in rat glomerular epithelial cells. Am J Pathol 1990;136:899-908.

21. Ichimura $K$, Powell $R$, Nakamura $T$ et al. Podocalyxin regulates pronephritic glomerular development in zebra fish. Physiol Rep 2013;1: e00074.

22. Takeda T, McQuistan T, Orlando RA et al. Loss of glomerular foot process is associated with uncoupling of podocalyxin from the actin cytoskeleton. J Clin Invest 2001;108:289-301.

23. Takenaka $Y$, Kanauchi M, Dohi $Y$ et al. Diagnostic significance of urinary sialic acid in diabetic nephropathy. Nihon Jinzo Gakkai Shi 1992;34: 85-90.

24. Yokoyama H, Jensen JS, Jensen $\mathrm{T}$ et al. Serum sialic acid concentration is elevated in IDDM especially in early diabetic nephropathy. J Int Med 1995;237:519-523.

25. Ozben T. Elevated serum and urine sialic acid levels in renal diseases. Ann Clin Biochem 1991;28:44-48.

26. Wang Z, Sun Z, Li AV et al. Roles for UDP-GlcNAc 2-epimerase/ManNAc 6-kinase outside of sialic acid biosynthesis: modulation of sialyltransferase and BiP expression, GM3 and GD3 biosynthesis, proliferation, and apoptosis, and ERK 1/2 phosphorylation. J Biol Chem 2006;281: 27016-27028.

27. Krause $S$, Hinderlich $S$, Amsili $S$ et al. Localisation of UDP-GICNAc 2epimerase/ManAc kinase (GNE) in the golgi complex and the nucleus of mammalian cells. Exp Cell Res 2005;304:365-379.

28. Zhu Y, Srivatana U, Ullah A. Suppression of a sialyltransferase by antisense DNA reduces invasiveness of human colon cancer cells in vitro. Biochim Biophys Acta 2001;1536:148-160.

29. Recchi MA, Hebbar M, Hornez $L$ et al. Multiplex reverse transcription polymerase chain reaction assessment of sialyltransferase expression in human breast cancer. Cancer Res 1998;58:4066-4070.

30. Pawluczyk IZA, Ghaderi-Najafabadi M, Patel $S$ et al. Sialic acid attenuates puromycin aminonucleoside-induced desialylation and oxidative stress in human podocytes. Exp Cell Res 2014;320:258-268.

31. Baricos WH, Cortez-Schwart S, Shah SV. Renal neuraminidase. J Biochem 1986;239:705-710.

32. Eguchi H, lkeda Y, Ookawara T et al. Modification of oligosaccharide by reactive oxygen species decreases sialyl lewis $x$-mediated cell adhesion. Glycobiology 2005;15:1094-1101.

33. Ginevri F, Gusmano R, Oleggini R. Renal purine efflux and xanthine oxidase activity during experimental nephrosis in rats: difference between puromycin aminonucleoside and Adriamycin nephrosis. Clin Sci 1990;78:283-293.

34. Ricardo SD, Bertram JF, Ryan GB. Reactive oxygen species in puromycin aminonucleoside nephrosis: in vitro studies. Kidney Int 1994;45:1057-1069.

35. Marshall CB, Pippin JW, Krofft RD et al. Puromycin aminonucleoside induces oxidant-dependent DNA damage in podocytes in vitro and in vivo. Kidney Int 2006;70:1962-1973.

36. Someya T, Kaneko K, Yamada T et al. Effect of free radical scavenger, edavarone, on puromucin aminonucleoside induced nephrosis in rats. Pediatr Nephrol 2005;20:1430-1434.

37. Ricardo SD, Bertram JF, Ryan GB. Podocyte architecture in puromycin aminonucleoside-treated rats administered tungsten or allopurinol. Exp Nephrol 1995;3:270-279.

38. Ames BN, Cathcart R, Schwiers E et al. Uric acid provides an antioxidant defence in humans against oxidant and radical-caused aging and cancer: a hypothesis. Proc Natl Acad Sci USA 1981;78:6858-6862.

39. Bedard K, Kause KH. The NOX family of ROS-generating NADPH oxidases: physiology and pathophysiology. Physiology Rev 2007;87: 245-313.

40. Rajendiran S, Lakshammanappa HS, Zachariah B et al. Desialylation of plasma proteins in severe dengue infection: possible role of oxidative stress. Am J Trop Med Hyg 2008;79:372-377.

41. Goswami K, Nandakumar DN, Koner BC et al. Oxidative changes in hyperthyroidism and desialylation of serum proteins in hyperthyroidism. Clin Chem Acta 2003;337:163-168.

42. Ito $M$, Sugihara $K$, Asaka $T$ et al. Glycoprotein hyposialylation gives rise to a nephrotc-like syndrome that is prevented by sialic acid administration in GNE V572L point-mutant mice. PLoS One 2012;7: e29873. 